\title{
Peri-operative care following left ventricular assist device implantation and heart transplantation
}

\begin{abstract}
With approximately 5.7 million people, in the United States alone, living with heart failure (HF), and less than 3,000 donor organs available for cardiac transplantation per year, the waiting period for heart transplantation (HTx) continues to increase. The implantation of a left ventricular assist device (LVAD) is now being used as not only a bridge to transplantation or recovery, but also as a destination therapy; it is important that they receive optimal care. By using identifiers for prolonged ventilation and markers for cardiac recovery, clinicians may be able to tailor specific treatment plans for these patients. The management of these specifically tailored plans can only be further optimized by continuous post-operative monitoring using trans esophageal echocardiography (TEE). They may choose to use the conventional post-operative care method, an unconventional one, or a combination of both but, as long as optimization of care is achieved for these patients, one can hope to see an increased survival rate and overall quality of life.
\end{abstract}

Volume 2 Issue 5 - 2015

\section{Christina Rocks BS}

Respiratory therapist, Long Island University, USA

Correspondence: Christina Rocks, Respiratory therapist, Long Island University - Brooklyn, 456 Beach 145th St Rockaway Park, NY I I694, USA, Tel 347-752-2992,

Email christina.rocks@my.liu.edu

Received: July 09, 2015 | Published: July 30, 2015
Abbreviations: TEE, trans esophageal echocardiography; $\mathrm{HF}$, heart failure; HT, heart transplantation; LVAD, left ventricular assist device; ESHF, end stage heart failure; LVHF, left ventricular heart failure; LV, left ventricular; LVEDV, left ventricular end diastolic volume; PV, prolonged ventilation; BNP, B-type natriuretic peptide; VAD, ventricular assist device; LVEDP, left ventricular end diastolic pressure; SBT, spontaneous breathing test; $\mathrm{CMV}$, continuous mechanical ventilation; MAP, mean arterial blood pressure; PAWP, pulmonary artery wedge pressures; PEEP, positive end expiratory pressure; RV, right ventricular; RR, respiratory rate; PAC, pulmonary artery catheter; PFO, patent foramen ovale; ICU, intensive care unit; ECMO, extracorporeal membrane oxygenation; BP, blood pressure; VV, venovenous; VA, venoarterial; PIP, peak inspiratory pressure; FIM, functional independence measure

\section{Introduction}

With approximately 5.7million people, in the United States alone, living with heart failure (HF), and less than 3,000 donor organs available for cardiac transplantation per year, the waiting period for heart transplantation (HTx) continues to increase. The implantation of a left ventricular assist device (LVAD) was originally used as a bridge to transplantation however, with the increased waiting period, an LVAD becomes a permanent or "destination" therapy for many HF patients. There is another group of HF patients which use LVADs as a bridge to recovery from cardiac failure. Compared with end stage heart failure (ESHF) patients receiving medical management, those who receive LVADs have improved survival rates and quality of life. ${ }^{1}$

\section{History}

Heart failure is a chronic condition characterized by the physiological consequence of impaired cardiac contractile function. When a patient is experiencing HF, compensatory mechanisms are called into action to maintain a sufficient blood pressure ensuring the perfusion of vital organs. Left ventricular heart failure (LVHF) is typically a result of impaired left ventricular (LV) contractility which means at a given preload, stroke volume is decreased resulting in an incomplete emptying of the ventricle. The combination of the "left-over" volume of blood in the ventricle, plus the volume from the next diastole leads to a stretch on the myofibers. This increased stretch stimulates a greater stroke volume on the next contraction aiding to empty the dilated ventricle and maintain cardiac output. This compensating action is known as the Frank-Starling mechanism. ${ }^{2}$ The position of the LV on the Frank-Starling curve is mainly determined by the left ventricular end diastolic volume (LVEDV); if enddiastolic volume falls, cardiac output also falls. Although it is initially beneficial, overtime the chronic rise in LV pressure and volume leads to myocardial hypertrophy, remodeling, and ultimately HF. ${ }^{3}$

\section{Left ventricular assist device vs. heart transplant}

While HTx is considered the gold standard for the treatment of $\mathrm{HF}^{4}{ }^{4}$ the lack of heart donors redirects most patient's plan of care towards LVAD implantation. A study examined respiratory gas analysis during exercise testing in patients with LVADs or who had had a HTx, both with or without a previous LVAD. Measurements taken to analyze the respiratory gas were heart rate, blood pressure, oxygen consumption $\left(\dot{\mathrm{V}}_{2}\right)$, carbon dioxide production $\left(\dot{\mathrm{VCO}}_{2}\right)$, minute ventilation $(\dot{\mathrm{V} E})$, and respiratory exchange ratio $\left(\mathrm{RQ}=\dot{\mathrm{V} C O} \mathrm{C}_{2} /\right.$ $\dot{\mathrm{V}} \mathrm{O}_{2}$ ).Patients were placed in sub-groups to compare exercise capacity at 8 and 12weeks after LVAD implantation, exercise performance at 12 weeks after LVAD implantation, exercise performance at 12 weeks and one year after HTx, and exercise capacity one year after HTx with and without a previous LVAD. Researchers found that patients in all sub-groups were able to eventually regain the same capacity for exercise as well as their exercise performance. This suggests that a permanent LVAD can be considered a final alternative to heart transplant. ${ }^{5}$ An LVAD is essentially a pump which is able to unload the left ventricle of the blood via an inflow cannula and pump it into the aorta through an outflow cannula. The LVAD is placed in the anterior mediastinum and is powered through a wire that exits in the body at the level of the abdomen and connects to a controller and energy source. ${ }^{6}$ Patients with LVHF presenting for LVAD implantation are 
typically hemodynamically fragile and display evidence of rapidly progressing end-organ dysfunction. ${ }^{7}$ Although it is ultimately the decision of the physician, whether or not a patient receives an LVAD, there are factors that help them arrive at that decision. The indications and contraindications to LVAD implantation are described in Table 1.

Table I The indications and contraindications to LVAD implantation ${ }^{4,8}$

\begin{tabular}{|c|c|}
\hline Indications to LVAD implantation & Contraindications to LVAD implantation \\
\hline $\begin{array}{l}\text { Left ventricular ejection fraction }<25 \% \text { and } \mathrm{VO} 2 \text { peak } \\
<12 \mathrm{ml} / \mathrm{kg} / \mathrm{min}\end{array}$ & $\begin{array}{l}\text { Anatomical issues (previous ventriculoplasty, previous mitral and/or aortic valve } \\
\text { replacement w/a mechanical prosthesis }\end{array}$ \\
\hline$\geq 3$ hospital admissions within the past year & Low body surface area (BSA) \\
\hline Dependence on inotropic support & Primary right ventricular dysfunction \\
\hline Pulmonary wedge pressure (PWP) $>20 \mathrm{mmHg}$ & Restrictive cardiomyopathy \\
\hline Systolic blood pressure (BP) $80-90 \mathrm{mmHg}$ & Significant ventricular arrhythmic pattern \\
\hline Cardiac index $(\mathrm{Cl}) \leq 2 \mathrm{~L} / \mathrm{min} / \mathrm{m}^{2}$ & Coagulopathy \\
\hline Mixed venous saturation (SVO2) $<60 \%$ & Sociocultural issues \\
\hline Initial right ventricular dysfunction & Hemodialysis \\
\hline
\end{tabular}

\section{Predictors of recovery}

Following LVAD implantation or HTx, many patients are successfully discharged home after only a brief hospital stay however, there are those who are not as fortunate. Some patients face postoperative complications which lead to prolonged hospital stay and eventually death. Most of these complications could be entirely avoided by taking the typical post-operative plan of care and tailoring it specifically to each individual patient to optimize their outcome. To aid in the optimization of patient care, identifying markers of cardiac recovery could help clinicians predict a patient's outcome to a certain treatment helping to better guide their plan.

\section{Prolonged ventilation}

It is known that following cardiac surgery, patients experiencing prolonged ventilation (PV) not only have a higher in-hospital mortality and morbidity but also have a poorer 5-year survival rate. An increasing amount of patients are facing these challenges due to their comorbidities and high-risk factors. ${ }^{9}$ A study aimed to recognize predictors of PV so these patients can be identified and undergo a patient-specific plan of treatment. The independent predictors of PV in cardiac surgery that this study identified include patients with

i. Increasing age $(\geq 65$ years old $)$

ii. Advanced New York Heart Association (NYHA) Class(III \& IV) [Refer to Table 2 for class description]

iii. Ejection fraction less than $50 \%$

iv. Renal dysfunction (creatinine $>200 \mu \mathrm{mol} / \mathrm{L}$ or dialysis)

v. Cardiopulmonary bypass (CPB) time more than 90minutes

vi. Multiple valve replacements

vii. Aortic procedures

viii. Reoperation for bleeding

ix. Need for inotropes or an intra-aortic balloon pump preoperatively

x. Categories based on how much they are limited during physical activity. ${ }^{10}$

xi. Although this may seem like a long list, researchers were also able to reject previous notions that the risk of PV was higher in patients with a history COPD, asthma, and smoking. By using this list of predictors for PV, these patients can be provided optimal clinical management both pre- and post-operatively. ${ }^{11}$

\section{Cardiac recovery}

In another attempt to identify predictive markers of cardiac recovery, researchers studied B-type natriuretic peptide (BNP). BNP is produced by cardiac ventricular myocytes in response to volume or pressure overload to decrease systemic vascular resistance, improve myocardial relaxation, increase natriuresis and suppress endothelin and the renin-angiotensin system. All of these factors lead to an increase in cardiac output, LVEDV, and left ventricular end diastolic pressure (LVEDP) which thereby worsen ventricular dysfunction, especially after cardiac surgery. Due to the fact that a critical cause of weaning failure is an underlying left ventricular dysfunction, this study aimed to determine whether or not BNP levels could be used to identify patients that would be difficult to wean from mechanical ventilation due to postoperative ventricular dysfunction after cardiac surgery. Compared to patients who were successfully weaned, they found that patients who failed to wean had higher BNP levels not only at their ICU admissions but also at the end of the spontaneous breathing test (SBT). This study showed that a strong predictor of (continuous mechanical ventilation) CMV weaning failure in patients after cardiac surgery is a high BNP concentration of $299 \mathrm{ng} / \mathrm{L}$ or higher at the end of the SBT. By monitoring BNP levels, clinicians can use this measurement as a guide and evaluation of therapies implemented prior to weaning so as to optimize ventricular function. ${ }^{12}$

Another study assessed the plasma levels of BNP in patients hospitalized for acutely decompensated chronic systolic heart failure both on their admission and discharge. They found that the BNP levels at discharge proved a stronger correlation with outcomes than admission levels however, both should be considered a significant prognostic factor in these patients. Over the course of the hospital stay, BNP levels are subject to change due to therapeutic interventions and therefore can be useful in determining a safe timing of discharge. The BNP cut-off values for long-term mortality were $1699 \mathrm{pg} / \mathrm{ml}$ on admission and $434.5 \mathrm{pg} / \mathrm{ml}$ at discharge; any values greater than or equal to these are a strong predictor of adverse outcomes. Using these cut-off values to identify higher risk patients will allow their treatment and discharge plans to be tailored more intensively than others. ${ }^{13}$ 
Table 2 The New York Heart Association (NYHA) Functional Classification places patients in one of four

\begin{tabular}{l} 
Class Functional capacity: how a patient with cardiac disease feels during physical activity \\
\hline I \\
Patients with cardiac disease but resulting in no limitation of physical activity. Ordinary physical activity does not cause undue \\
fatigue, palpitation, dyspnea or anginal pain. \\
Patients with cardiac disease resulting in slight limitation of physical activity. They are comfortable at rest. Ordinary physical \\
activity results in fatigue, palpitation, dyspnea or anginal pain. \\
Patients with cardiac disease resulting in marked limitation of physical activity. They are comfortable at rest. Less than ordinary \\
activity causes fatigue, palpitation, dyspnea or anginal pain. \\
Patients with cardiac disease resulting in inability to carry on any physical activity without discomfort. Symptoms of heart failure \\
or the anginal syndrome may be present even at rest. If any physical activity is undertaken, discomfort increases
\end{tabular}

\section{Post-operative management}

Following ventricular assist device (VAD) implantation, and observing conventional post-operative care, most patients should be able to be weaned from mechanical ventilation and woken within a few hours, providing that they remain hemodynamically stable and meet CMV weaning criteria. To ensure a patient's hemodynamic stability during the early postoperative period, they must maintain a normal sinus rhythm, mean arterial blood pressure (MAP) of 60$80 \mathrm{mmHg}$, central venous (CVP) and pulmonary artery wedge (PAWP) pressures less than $15 \mathrm{mmHg}$ and a cardiac index above $2.51 \mathrm{~min}^{-}$ ${ }^{1} \mathrm{~m}^{-2}$. Although CMV weaning criteria can differ depending on the institution, recommended criteria includes $\mathrm{pH} \geq 7.25$, partial pressure of oxygen in the arterial blood $\left(\mathrm{PaO}_{2}\right) \geq 60 \mathrm{mmHg}$, fraction of inspired oxygen $\left(\mathrm{FIO}_{2}\right) \leq 0.4, \mathrm{PaO}_{2} / \mathrm{FIO}_{2}(\mathrm{P} / \mathrm{F})$ ratio $\geq 150 \mathrm{mmHg}$, positive end expiratory pressure (PEEP) $\leq 8 \mathrm{cmH}_{2} \mathrm{O}$, and the patient's capability of triggering the ventilator. ${ }^{14}$

\section{Ventilator settings}

A common complication faced after LVAD implantation is right ventricular (RV) failure. This can be avoided by titrating fluids and LVAD settings to maintain the ventricular septum in a neutral position and support RV systolic function. Respiratory complications can be avoided by early extubation and mobilization however, when prolonged $\mathrm{CMV}$ is indicated, routine ventilator settings should be followed. These settings are tidal volume $(\mathrm{Vt})$ of $6-8 \mathrm{ml} / \mathrm{kg}$, PEEP of $5 \mathrm{cmH}_{2} \mathrm{O}$, respiratory rate $(\mathrm{RR})$ adjusted to maintain partial pressure of carbon dioxide in the arterial blood $\left(\mathrm{PaCO}_{2}\right)$ between 35 and $45 \mathrm{mmHg}$, and $\mathrm{FIO}$, adjusted to maintain $\mathrm{PaO}_{2}$ above $75 \mathrm{mmHg}$ and oxygen saturation $\left(\mathrm{SpO}_{2}\right)>92 \%$. By optimizing these conditions in patients, one can hope to help a patient recover smoothly and swiftly following VAD implantation. ${ }^{15}$

\section{Monitoring}

For the post-operative management of cardiac surgery patients, the pulmonary artery catheter (PAC) has remained the "gold standard." Used in conjunction with other monitors like an arterial pulse waveform analysis and Doppler analysis of descending aortic flow, the PAC aids clinicians in managing fluids and vasoactive agents. Although effective, these monitors have several limitations, most notably, the inability to diagnose major pathology. This is not to say that adverse outcomes can be blamed on the monitor alone; it is the way one chooses to manage changes based on the information gathered by the monitor which is responsible for the course of action.

\section{Echocardiography}

Although other forms of postoperative cardiac monitoring may be able to identify low cardiac output, hypotension, hypoperfusion, and lactatemia, they cannot always identify the cause. Pericardial hematoma and tamponade, vasodilatory shock, hemorrhage, hypovolemia, LV and RV failure are all common postoperative complications that require immediate diagnosis and treatment. If not promptly treated, patients who suffer from these complications risk not only a longer stay in critical care, but also end organ failure and procedural mortality. Echocardiography provides not only qualitative diagnostic information but also quantitative dimensional and Doppler gathered data relating to hemodynamics and cardiac function. The use of early echocardiography can be used to diagnose or at the very least, rule out one of the aforementioned complications. Specifically, trans esophageal echocardiography (TEE) can be used to diagnose a right-to-left intracardiac shunt such as a patent foramen ovale (PFO); a common cause of severe hypoxemia mimicking lung failure after VAD implantation. In recent cases, it has been integrated and used for routine post-operative cardiac surgery management of fluids, inotropes, ventilator settings, and mechanical support. ${ }^{16}$

A case report presents a 62 year old patient admitted to the cardiac intensive care unit (ICU) less than 24hours after an anterior STsegment elevated myocardial infarction. Echocardiography revealed a $15 \%$ LV ejection fraction which required emergency coronary angiography, revascularization, and insertion of an intra aortic balloon pump. After thromboaspiration, a stent was placed to secure the left anterior descending artery which was totally occluded. Despite these interventions, the patient remained hemodynamically unstable and required the placement of an LVAD as rescue therapy. Several minutes after the implantation of the LVAD, severe hypoxia occurred requiring the $\mathrm{FIO}_{2}$ to be raised from 0.4 to 1.0 with a $\mathrm{P} / \mathrm{F}$ ratio of 59 . There was no change in lung auscultation and chest $\mathrm{x}$-rays revealed only moderate edema. Pulmonary compliance was measured as $36 \mathrm{~mL} /$ $\mathrm{cmH}_{2} \mathrm{O}$. While leaving hemodynamic parameters unchanged with a $3-\mathrm{L} / \mathrm{min}$ LVAD output and increasing PEEP from 5 to $10 \mathrm{cmH}_{2} \mathrm{O}$, there was a decrease in $\mathrm{P} / \mathrm{F}$ ratio. When the LVAD output was increased from 3.0- to 3.5-L/min, the P/F ratio decreased even further. Due to the suspicion of a PFO, PEEP and LVAD outflow were reduced which led to an immediate improvement and blood oxygenation normalization.

The supposed cause of the shunt was the combination of the LV unloading by the LVAD as well as the increased RV after load, external factors opposing muscle fiber shortening, ${ }^{17}$ by PEEP. To confirm this suspicion, a TEE micro bubbles test was performed with different PEEP and LVAD output settings. They found that as the LVAD output and PEEP increased, the shunt proportionally increased in size. TEE was used to adjust the PEEP and LVAD output levels to control the right-to-left shunt with $2-\mathrm{cmH}_{2} \mathrm{O}$ and $3-\mathrm{L} / \mathrm{min}$ respectively. For the following 3days, TEE was used to continue managing hemodynamic 
and respiratory support. As pulmonary compliance improved, PEEP was safely increased under TEE control without inducing the right-toleft shunt. PEEP is tricky to set in these situations due to the heart's sensitivity to the changes in trans pulmonary pressure. An increase in PEEP is able to decrease LV after load and oxygen consumption while also enhancing cardiac output. Unfortunately, that increase in PEEP also results in an increase in RV after load and subsequently, a rightto-left intra arterial shunt. ${ }^{18}$ Although not all patients will present with PFO following LVAD implantation, TEE should still be performed routinely to help manage LVAD output and PEEP pressure settings.

Continuous or semi-continuous echocardiography monitoring is now available thanks to the development of a new, miniaturized disposable TEE probe with an indwelling time of 72 hours. ${ }^{19}$ By the LVAD unloading the LV, the RV after load is passively increased therefore, with the addition of excess PEEP even more RV dilation occurs. TEE can be used in this situation to optimize preload reduction, inotropic titration, and after load reduction using pulmonary vasodilators. The idea of routine assessment by echocardiography in the ICU is goal directed rather than the traditional use in crisis management. The successful integration of TEE with other monitors in the cardiac surgical critical care setting can aid in the understanding of physiologic changes, optimization of therapies, and thereby improve patient outcomes. ${ }^{14}$

An observational study conducted in a cardiovascular ICU used the new, miniature TEE probe, comparable size to the size of a nasogastric tube, to monitor high-risk cardiac surgery patients. The researchers hypothesized that episodic monoplane TEE guides the assessment of intravascular/myocardial volume, inotrope need, vasopressor use, and assessment of pericardial effusions in these critically ill patients. Following cardiac surgery, if any patient became hemodynamically unstable in the ICU, a monoplane TEE was introduced. Patients were considered hemodynamically unstable if they had a persistent systolic blood pressure (BP) $<100 \mathrm{mmHg}$, cardiac index $<2.2 \mathrm{~L} / \mathrm{min} / \mathrm{m}^{2}$, mixed venous oxygen saturation $(\mathrm{SvO} 2)<60 \%$, suspected pericardial effusion with tamponade physiology, base deficit $>8 \mathrm{mEq} / \mathrm{L}$, or lactate $>5 \mathrm{mg} / \mathrm{dL}$ that was unresponsive to inotropic, vasopressor, and/or volume resuscitation.

Following TEE placement, researchers performed an imaging session every 2 to 3 hours for the first 6hours and continued as needed until the patient either reached hemodynamic stability or the 72 hours post-operative mark. The focus of their assessment was on the LV end-diastolic area, LV fractional area change, RV function, intravascular volume status, and responsiveness to fluid resuscitation and pericardial effusion with tamponade physiology. If at any time TEE findings convinced the clinician to change management direction from what was thought before the TEE exam, it was considered hemodynamic discordance. In this study, discordance between standard hemodynamic monitoring and episodic limited TEE was observed in $66 \%$ of patients. Although the direct impact of episodic monoplane TEE on patient outcome in the cardiac surgery ICU has yet to be determined, this study presented its ability to elucidate physiologic derangement and guide therapy. Some may shy away from episodic echocardiography due to its added expense however, these patients are already so financially invested that if this could prevent them from having to return to the operating room and avoid further complications, most are willing to do it. ${ }^{20}$

\section{Extracorporeal membrane oxygenation}

When conventional mechanical ventilation fails and patients experience pulmonary and/or cardiac failure, they may be supported by extracorporeal membrane oxygenation (ECMO). ECMO is a temporary form of life support which provides a prolonged biventricular circulatory and pulmonary support. ${ }^{21}$ When a patient is placed on ECMO, their blood is drained from the venous system, pumped through an oxygenator, and then re-infused to the patient. For patients only receiving respiratory support, venovenous (VV) ECMO is used while patients requiring both respiratory and circulatory support receive venoarterial (VA) ECMO to bypass both the heart and lungs. Bi-level positive airway pressure is continued throughout the ECMO procedure with the typical "rest" ventilator settings at a peak inspiratory pressure (PIP) $20-25 \mathrm{cmH}_{2} \mathrm{O}, \mathrm{PEEP} 10-15 \mathrm{cmH}_{2} \mathrm{O}, \mathrm{FIO}_{2}$ less than 0.5 , RR of $4-8 \mathrm{bpm}$, and a tidal volume less than $100 \mathrm{~mL}$.

Since patients who have VAD implants are only receiving circulatory support, ECMO may be indicated to assist in oxygenating and ventilating, especially in those patients sick with pulmonary edema-associated cardiomyopathy. ECMO can prove to be extremely beneficial in oxygenating and ventilating these cardiac or circulatory impaired patients without exposing them to the risk of ventilator induced lung injuries. ${ }^{22}$

\section{Exercise rehabilitation}

It is important that, early on, following CMV liberation, an exercise program is initiated. An early initiation of a structured exercise program for patients with newly implanted LVADs has been shown to not only improve exercise capacity but also survival rate. A study used subjects classified as having NYHA classes of III or IV heart failure pre-implantation. After implantation, the patients began their acute inpatient rehabilitation of 3hours of therapy daily for 6 days per week. All of the patients were observed to have improved peak oxygen consumption levels, systemic vascular resistance, and mean pulmonary arterial pressure after exercise. The success of their rehabilitation was based on functional independence measure (FIM) gain and efficiency. The FIM system measures progress in activities of daily living, mobility, and communication. In this study, LVAD patients had a mean FIM gain of 28.6 and efficiency of 1.97, better than that of the national FIM gain of 23 and efficiency of 2.28. They all safely tolerated their regimens, displaying a considerable functional benefit from it and experienced fewer LVAD-related complications.1There are other studies that show that patients with LVADs for long-term support are able to increase LVAD output and potentially the patient's own cardiac output through exercise. ${ }^{23}$ It is believed that these increases are due to improved peripheral circulation. To accommodate growing populations of cardiac care patients, in-patient rehabilitation units should strongly consider implementing these more aggressive exercise rehabilitation programs.

\section{Summary}

When treating patients, clinicians "shoot for the good without creating the bad" however, it is unreasonable to think that one treatment plan will be beneficial to every patient because as we know, no two patients are exactly alike. It is using this logic that inspires researchers to identify alternative therapies and treatments that adhere to each individual patient's needs while producing a sufficient enough response. Due to the fact that LVAD implantation is becoming a destination therapy for so many patients, it is important that they receive optimal care. By using identifiers for prolonged ventilation and markers for cardiac recovery, clinicians may be able to tailor specific treatment plans for these patients. The management of these specifically tailored plans can only be further optimized by continuous post-operative monitoring. They may choose to use the conventional post-operative care method, an unconventional one, or a combination 
of both but, as long as optimization of care is achieved for these patients, one can hope to see an increased survival rate and overall quality of life.

\section{Acknowledgements}

None.

\section{Conflict of interest}

The author declares no conflict of interest. 